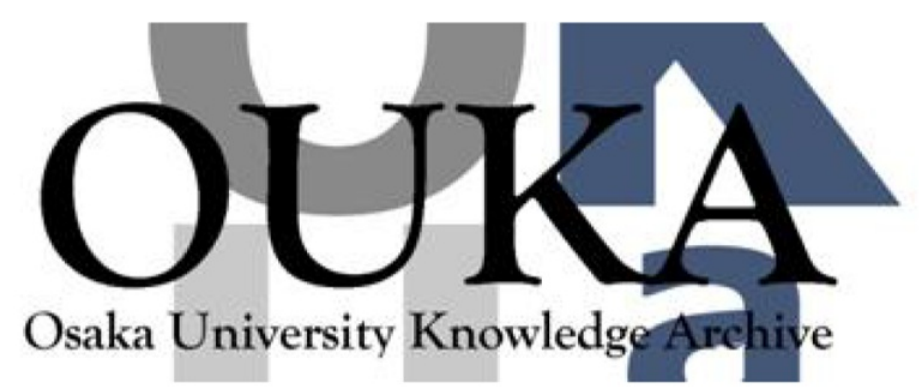

\begin{tabular}{|c|l|}
\hline Title & $\begin{array}{l}\text { Unusual elastic behavior of nanocrystalline } \\
\text { diamond thin films }\end{array}$ \\
\hline Author(s) & Tanei, H.; Nakamura, N.; Ogi, H. et al. \\
\hline Citation & $\begin{array}{l}\text { Physical Review Letters. 100(1) p. 016804-1- } \\
\text { p.016804-4 }\end{array}$ \\
\hline Issue Date & $2008-01-11$ \\
\hline oaire:version & VoR \\
\hline URL & https://hdl. handle. net/11094/84181 \\
\hline rights & Copyright 2008 by the American Physical Society \\
\hline Note & \\
\hline
\end{tabular}

Osaka University Knowledge Archive : OUKA

https://ir. Library. osaka-u. ac. jp/

Osaka University 


\title{
Unusual Elastic Behavior of Nanocrystalline Diamond Thin Films
}

\author{
H. Tanei, N. Nakamura, H. Ogi, and M. Hirao \\ Graduate School of Engineering Science, Osaka University, Toyonaka, Osaka 560-8531, Japan
}

\author{
R. Ikeda
}

Research and Development, Asahi Diamond Industrial Co., Ltd., 787 Tabi, Ichihara, Chiba 290-0515, Japan

(Received 13 July 2007; published 11 January 2008)

\begin{abstract}
This Letter studies the relationship between the elastic constants and the microstructure of nanocrystalline diamond thin films deposited by the chemical vapor deposition method doping various concentration of $\mathrm{N}_{2}$ gas. The elastic constants were measured by resonant ultrasound spectroscopy and picosecond laser ultrasounds. The increase of $\mathrm{N}_{2}$ gas decreases the diagonal elastic constants, but increases the off-diagonal elastic constants. The micromechanics calculation can explain this unusual elastic behavior, and it predicts thin graphitic phases at grain boundaries.
\end{abstract}

The diamond thin films deposited by the chemical vapor deposition (CVD) technique are prospective materials for improvements of many applications because of their outstanding physical properties. However, they have rough surfaces, making it difficult to apply them to acoustic devices. Recently, nanocrystalline diamond (NCD) thin films were synthesized by incorporating nitrogen gas, which restricted growth of crystallites and reduced the grain size to be of the order of nanometers [1-3]. The NCD films show highly smooth surfaces, while the nitrogen gas deteriorates the attractive properties of diamond such as high hardness and high stiffness.

The microstructure of NCD film has been studied by transmission electron microscopy [1-4], Raman spectroscopy [4-6], and electron energy-loss spectroscopy (EELS) $[1,3]$. These works suggest that the NCD films contain a small amount of $s p^{2}$-bonded carbons near boundaries of $s p^{3}$-bonded crystal-carbon grains [3-6]. However, details of the structure of the $s p^{2}$-bonded region and its influence on the mechanical properties remain unclear.

We here evaluate the microstructure of NCD thin films via the elastic constants. The elastic constants of thin films are sensitive to defects, incohesive bonded regions, as well as impurities. Polycrystalline thin films macroscopically show transverse isotropy about the film-growth axis, and they have five independent macroscopic elastic constants $\left(C_{11}, C_{33}, C_{12}, C_{13}\right.$, and $\left.C_{44}\right)$ when the $x_{3}$ axis is along the thickness direction [7]. The accurate measurement of the elastic constants enables us to evaluate the microstructure of films because the inclusions affect the stiffness and elastic anisotropy. However, the conventional techniques for measuring the elastic constants based on microtensile tests [8,9], nanoindentation [10], and reed vibrations $[11,12]$ fail to determine the anisotropic elastic constants of thin films because of the large effects of the dimension errors of the specimen and ambiguous gripping conditions. In order to determine the elastic constants of thin films accurately, we developed resonant ultrasound spectroscopy coupled with laser-Doppler interferometry (RUS-LDI) [13-16]. With this method, we measured the elastic constants of CVD diamond thin films and found that the diagonal elastic constants $C_{11}$ and $C_{66}\left[=\left(C_{11}-C_{12}\right) / 2\right]$ of NCD films were smaller than those of microcrystalline diamond thin films, while the off-diagonal elastic constant $C_{12}$ of NCD film was larger $[15,16]$. In this Letter, we elucidate the origins of this unusual elastic behavior of the NCD thin films. For this purpose, we developed the picosecond laser ultrasounds (PSLU) method [17-21] to measure the out-of-plane elastic modulus $C_{33}$, because it is necessary for accurately determining the in-plane elastic constants by the RUS-LDI method through the inverse calculation.

We prepared three kinds of NCD films by the hotfilament CVD method on monocrystal silicon. The source gases were $\mathrm{CH}_{4}, \mathrm{H}_{2}$, and $\mathrm{N}_{2} . \mathrm{CH}_{4}$ and $\mathrm{H}_{2}$ flow rates were 6 and $200 \mathrm{sccm}$, respectively, and concentrations of $\mathrm{N}_{2}$ to the whole source gases were $0 \%, 0.2 \%$, and $0.5 \%$ (the unit sccm stands for standard cubic centimeters per minute, i.e., $\mathrm{cm}^{3} / \mathrm{min}$ at $0^{\circ} \mathrm{C}$ in $\left.1 \mathrm{~atm}\right)$. The temperature of the substrate during deposition was $770^{\circ} \mathrm{C}$. The film thicknesses were between 11 and $15 \mu \mathrm{m}$, determined by field-emission scanning electron microscopy (FE-SEM) on the cross sections. Figure 1 shows their surface morphology and corresponding Raman spectra. The grain size decreases with the increase of the $\mathrm{N}_{2}$-gas concentration from $\sim 5 \mu \mathrm{m}$ to $\sim 5 \mathrm{~nm}$. The Raman spectra show the sharp peak at $1330 \mathrm{~cm}^{-1}$, which corresponds to the $s p^{3}$-bonded region of the specimen. This peak becomes broader, and a much broader peak appears at around $1500 \mathrm{~cm}^{-1}$ for the specimens deposited with $\mathrm{N}_{2}$ gas. This broad peak consists of various $s p^{2}$ compounds, including the amorphous phase and the graphite phase [4], and it is difficult to identify the structure and their contents only from the peaks. However, it indicates that $\mathrm{N}_{2}$ gas generates the $s p^{2}$-bonded regions.

The RUS-LDI method uses the free-vibration resonant frequencies of the film or substrate specimen, which de- 

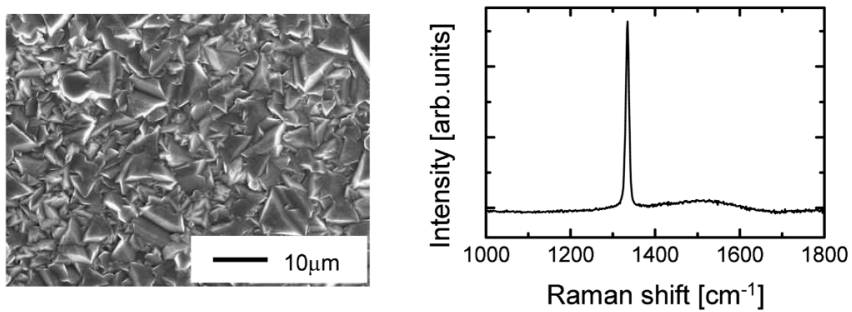

(a) $\mathrm{N}_{2}: 0 \%$
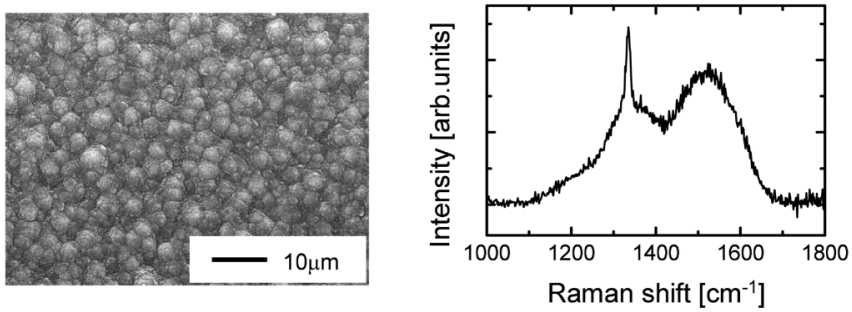

(b) $\mathrm{N}_{2}: 0.2 \%$
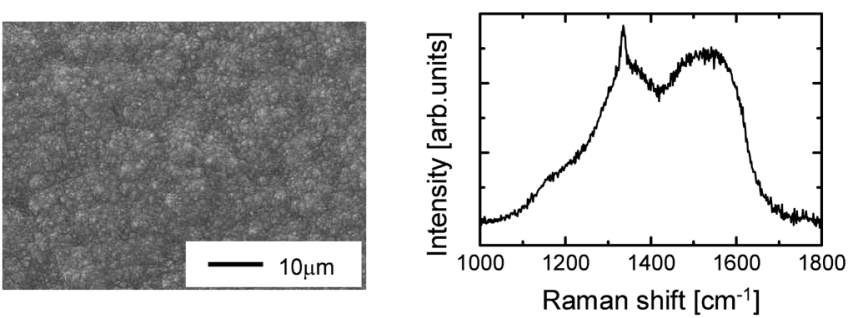

(c) $\mathrm{N}_{2}: 0.5 \%$

FIG. 1. SEM images of surface morphology (left) and Raman spectra (right).

pend on the dimensions, mass density, and all independent elastic constants of the film and the substrate. The elastic constants of the film can be determined inversely by measuring other parameters. In order to determine the elastic constants, identification of the vibrating mode is indispensable. This process was carried out with laser-Doppler interferometry (LDI), which measured the distributions of the out-of-plane displacement of the vibrating specimen. The details of the RUS-LDI method are given in [13-16]. The elastic constants $C_{33}$ and $C_{44}$ of the film cannot be determined accurately with this method, because their contributions to the resonant frequencies are too small. The contribution $\left(\frac{\partial f}{\partial C_{i j}}\right) \frac{C_{i j}}{f}$ of $C_{44}$ is about $10^{-6}$ and that of $C_{33}$ is about $10^{-3}$. Thus, we can neglect the contribution of $C_{44}$. We instead determined the out-of-plane modulus $C_{33}$ by the PSLU method to have the full set of moduli other than $C_{44}$.

We deposited a 10-nm Al film on the diamond films for PSLU. A pump light (wavelength, power, and duration are $800 \mathrm{~nm}, 25 \mathrm{~mW}$, and $100 \mathrm{fs}$, respectively) irradiates the Al surface to generate the longitudinal waves in the diamond film through the thermal expansion of the Al film. Then, a time-delayed probe light $(400 \mathrm{~nm}, 15 \mathrm{~mW}$, and $100 \mathrm{fs}$ ) is normally applied to the surface, which is diffracted by the longitudinal wave propagating in the thickness direction in the diamond film. The diffracted light becomes coherent when the wavelength of the longitudinal wave equals $\lambda_{o} / 2 n$ (Bragg diffraction), where $\lambda_{o}$ and $n$ represent the wavelength of the probe light and the refractive index of diamond, respectively $[17,18,22]$. The diffracted light interferes with the reflected light at the Al surface, which causes oscillations in the intensity response of the reflected probe light as the longitudinal wave travels. The period of the oscillation gives the frequency of the longitudinal wave $f$, and the elastic constant $C_{33}$ is determined from $C_{33}=$ $\rho\left(f \lambda_{o} / 2 n\right)^{2}$. Details of our optics are shown in $[19,20]$. Figure 2 shows the oscillations of the reflectivity of the probe light and the corresponding Fourier spectra. Increase of $\mathrm{N}_{2}$ gas decreases the frequency, indicating the decrease of $C_{33}$. We used $\rho=3516 \mathrm{~kg} / \mathrm{m}^{3}$ [23] for all specimens, which is the value of bulk diamond, and $n=2.470,2.450$, and 2.436 for the specimens deposited with $0 \%, 0.2 \%$, and $0.5 \% \mathrm{~N}_{2}$ gas, respectively. The indices were measured by the ellipsometry measurements [24]. The determined $C_{33}$ by the PSLU method was used to obtain the other elastic constants in the RUS-LDI method. Details of the RUS-LDI measurement for the present specimens appear in the supplemental file [24].

Figure 3 shows the determined elastic constants of the NCD films. The broken lines represent the elastic constants of isotropic bulk diamond (no defects), which are calculated by the Hill averaging approximation [25]. We note the following important observations: (i) The increase of $\mathrm{N}_{2}$ gas decreases the diagonal elastic constants, which is expected, but (ii) it increases the off-diagonal elastic constants $C_{12}$ and $C_{13}$. As the increase of $\mathrm{N}_{2}$ gas reduces the grain size, the volume fraction of the grain boundaries
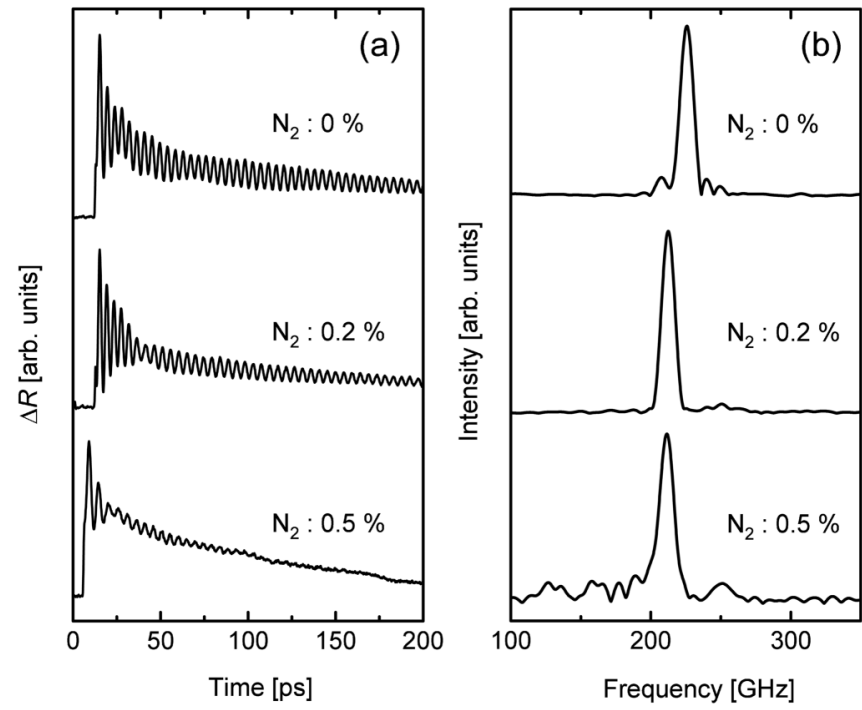

FIG. 2. (a) Observed reflectivity changes from NCD films and (b) their Fourier spectra. The frequency decreases as the $\mathrm{N}_{2}$ flow rate increases. 

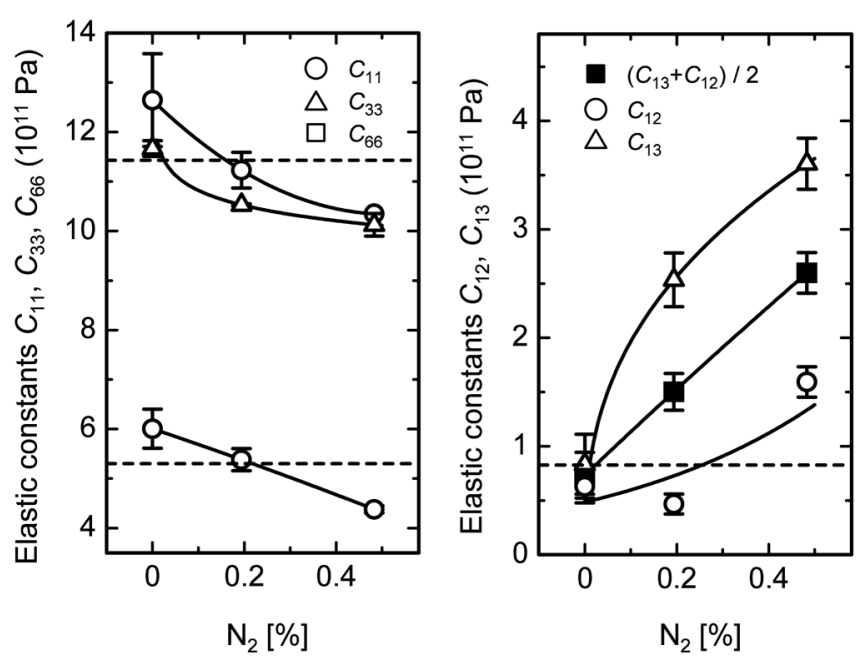

FIG. 3. $\mathrm{N}_{2}$-flow-rate dependence of (left) diagonal elastic constants $C_{11}, C_{33}$, and $C_{66}$, and (right) off-diagonal elastic constants $C_{12}$ and $C_{13}$. Broken lines represent the elastic constants of the isotropic bulk diamond. The error bar mainly results from an inaccuracy of the film-thickness determination.

increases, to which we attribute this unusual elastic behavior.

Raman spectra show that the doped $\mathrm{N}_{2}$ gas develops the $s p^{2}$-bonded carbons. Raman spectroscopy and EELS studies [3-6] indicate that the NCD films consist of $s p^{3}$ grains and $s p^{2}$-bonded grain boundaries. We then investigate the influence of $s p^{2}$-bonded carbons at grain boundaries on the elastic constants of NCD films using micromechanics calculation [26]. For this, we consider a model that the NCD film consists of $s p^{3}$-bonded carbons and $s p^{2}$-bonded thinplate inclusions to simulate the above microstructure. The elastic constants were obtained by the following procedures: (i) Calculation of the elastic constants of the composite consisting of an isotropic diamond matrix and oriented pancake-shaped inclusions. This composite has elastically hexagonal symmetry. (ii) Calculation of the direction-over-averaged elastic constants of the structure obtained in (i), simulating randomly oriented inclusions embedded in the diamond matrix. Then, this composite shows elastically isotropic. Further details of the procedure appear in the supplemental file [24]. As the inclusions, we assume the following four cases: (a) monocrystal graphite thin plates (typical $s p^{2}$ structure), whose $c$ axes are along the minor axes (along the normal direction to the grain boundary), (b) monocrystal graphite thin plates whose $a$ axes are along the minor axes, (c) diamondlike carbons (DLC), and (d) microcracks (voids). The elastic constants of these isotropic composites were calculated varying the volume fraction of inclusions, assuming various aspect ratios. We referred to reported values as the elastic constants of monocrystal graphite [27] and DLC [28], respectively.

Figure 4 plots the elastic constants of the composites relative to those of the isotropic diamond matrix (no in-

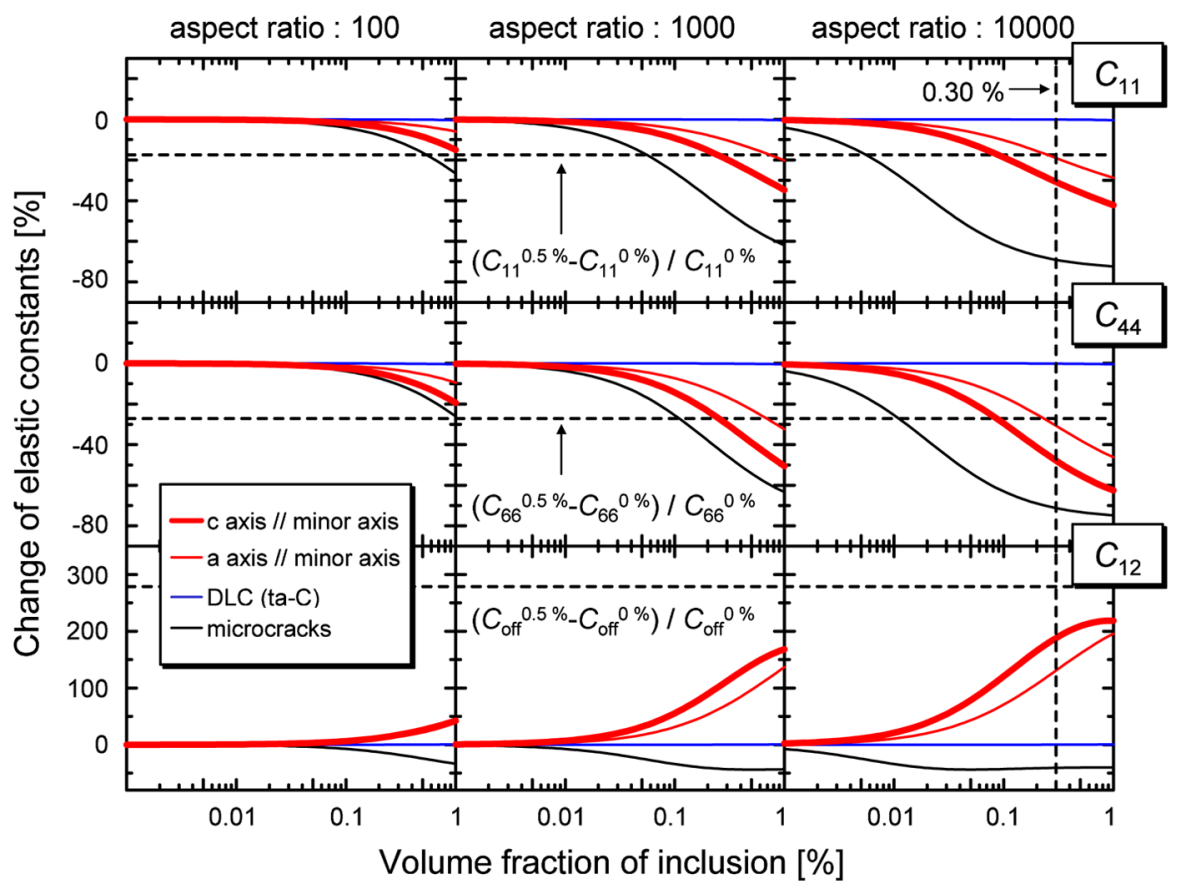

FIG. 4 (color online). The changes of the elastic constants of the composites including various inclusions for the aspect ratio of the thin-plate inclusion of 100,1000, and 10000 . Only the monocrystal graphite plate inclusions cause the opposite behaviors between the diagonal and off-diagonal elastic constants. Broken horizontal lines represent the percentage of measured elastic constants of the NCD film deposited with $0.5 \% \mathrm{~N}_{2}$ gas to that with $0 \% \mathrm{~N}_{2}$ gas, and the vertical broken line represents the estimated volume fraction of the monocrystal graphitic inclusion. 
clusions) for the aspect ratios of the inclusions of 100 , 1000 , and 10000 . When the inclusions are microcracks, all elastic constants decrease with the increase of the volume fraction. DLC inclusions scarcely change the elastic constants. Thus, microcracks and DLC in grain boundaries cannot explain the measurements. On the other hand, the graphitic inclusions, especially when their $c$ axes are along the minor axes of inclusions, increase $C_{12}$ drastically, which is consistent with the measurement. Therefore, such a microstructure that consists of $s p^{3}$-diamond grains and monocrystal graphitic phases ( $s p^{2}$-bonded carbons) at grain boundaries is most reasonable. Furthermore, we can estimate the volume fraction of the graphite inclusions quantitatively. In Fig. 4, the horizontal broken lines represent the percentage of measured elastic constants of NCD film deposited with $0.5 \% \mathrm{~N}_{2}$ gas to that with $0 \% \mathrm{~N}_{2}$ gas $\left[C_{\text {off }}\right.$ in the bottom figure denotes the average of the offdiagonal elastic constants: $\left(C_{12}+C_{13}\right) / 2$ ]. The best fitting volume fraction of inclusion is about $0.30 \%$, which is shown with the vertical broken line in Fig. 4, when we assume the $c$ axes of the graphite inclusions are normal to the $s p^{3}$ diamond grain boundaries and the aspect ratio of the thin plate is 10000 .

In summary, the anisotropic elastic constants of NCD films deposited with $\mathrm{N}_{2}$-gas doping were determined using RUS-LDI and PSLU methods. As $\mathrm{N}_{2}$ gas increases, the diagonal elastic constants $C_{11}, C_{33}$, and $C_{66}$ decrease, while the off-diagonal elastic constants $C_{12}$ and $C_{13}$ increase. Considering micromechanics calculation, such a result can be explained only when the monocrystalgraphitic-plate inclusions are distributed among diamond grains. Thus, it was indicated that the NCD films consist of $s p^{3}$-diamond grains and monocrystal graphitic phases in grain boundaries. Our measurement and calculation have shown the potential to determine the volume fraction of inclusion quantitatively.

[1] D. Zhou, A. R. Krauss, L. C. Qin, T. G. McCauley, D. M. Gruen, T.D. Corrigan, R. P.H. Chang, and H. Gnaser, J. Appl. Phys. 82, 4546 (1997).

[2] S. Bhattacharyya, O. Auciello, J. Birrell, J. A. Carlisle, L. A. Curtiss, A. N. Goyette, D. M. Gruen, A. R. Krauss, J. Schlueter, A. Sumant, and P. Zapol, Appl. Phys. Lett. 79, 1441 (2001).

[3] J. Birrell, J. A. Carlisle, O. Auciello, D. M. Gruen, and J. M. Gibson, Appl. Phys. Lett. 81, 2235 (2002).
[4] J. Birrell, J.E. Gerbi, O. Auciello, J. M. Gibson, J. Johnson, and J. A. Carlisle, Diam. Relat. Mater. 14, 86 (2005).

[5] A.C. Ferrari and J. Robertson, Phys. Rev. B 61, 14095 (2000).

[6] J. Birrell, J.E. Gerbi, O. Auciello, J.M. Gibson, D. M. Gruen, and J. A. Carlisle, J. Appl. Phys. 93, 5606 (2003).

[7] N. Nakamura, H. Ogi, T. Ichitsubo, and M. Hirao, J. Appl. Phys. 94, 6405 (2003).

[8] W. Suwito, M. L. Dunn, S. J. Cunningham, and D. T. Read, J. Appl. Phys. 85, 3519 (1999).

[9] H. Huang and F. Spaepen, Acta Mater. 48, 3261 (2000).

[10] T. Malkow, I. A. García, A. Kolitsch, D. Schneider, S. J. Bull, and T. F. Page, Diam. Relat. Mater. 10, 2199 (2001).

[11] H. Mizubayashi, J. Matsumoto, and H. Tanimoto, Scr. Mater. 41, 443 (1999).

[12] S. Sakai, H. Tanimoto, and H. Mizubayashi, Acta Mater. 47, 211 (1999).

[13] H. Ogi, K. Sato, T. Asada, and M. Hirao, J. Acoust. Soc. Am. 112, 2553 (2002).

[14] N. Nakamura, H. Ogi, and M. Hirao, Acta Mater. 52, 765 (2004).

[15] H. Ogi, N. Nakamura, H. Tanei, M. Hirao, R. Ikeda, and M. Takemoto, Appl. Phys. Lett. 86, 231904 (2005).

[16] R. Ikeda, H. Tanei, N. Nakamura, H. Ogi, M. Hirao, A. Sawabe, and M. Takemoto, Diam. Relat. Mater. 15, 729 (2006).

[17] C. Thomsen, J. Strait, Z. Vardeny, H. J. Maris, J. Tauc, and J. J. Hauser, Phys. Rev. Lett. 53, 989 (1984).

[18] C. Thomsen, H. T. Grahn, H. J. Maris, and J. Tauc, Phys. Rev. B 34, 4129 (1986).

[19] H. Ogi, M. Fujii, N. Nakamura, T. Shagawa, and M. Hirao, Appl. Phys. Lett. 90, 191906 (2007).

[20] H. Ogi, M. Fujii, N. Nakamura, T. Yasui, and M. Hirao, Phys. Rev. Lett. 98, 195503 (2007).

[21] N. Nakamura, H. Ogi, T. Yasui, M. Fujii, and M. Hirao, Phys. Rev. Lett. 99, 035502 (2007).

[22] A. Devos and R. Côte, Phys. Rev. B 70, 125208 (2004).

[23] H. J. McSkimin and W.L. Bond, Phys. Rev. 105, 116 (1957).

[24] See EPAPS Document No. E-PRLTAO-100-052802 for further details. For more information on EPAPS, see http:// www.aip.org/pubservs/epaps.html.

[25] R. Hill, J. Mech. Phys. Solids 5, 229 (1957).

[26] M.L. Dunn and H. Ledbetter, Acta Mater. 45, 3327 (1997).

[27] O. L. Blakslee, D. G. Proctor, E. J. Seldin, G. B. Spence, and T. Weng, J. Appl. Phys. 41, 3373 (1970).

[28] R. Pastorelli, A. C. Ferrari, M. G. Beghi, C. E. Bottani, and J. Robertson, Diam. Relat. Mater. 9, 825 (2000). 